\title{
Statins role in preventing contrast-induced acute kidney injury: a scoping review
}

\author{
Ibrar Anjum *, Manahil Akmal, Nimra Hasnain, Maha Jahangir, Wafa Sohail
}

\section{A B S T R A C T}

Background: Acute renal failure secondary to contrast-induced acute kidney injury (CI-AKI) is one of the most commonly encountered problems in hospitalised patients. The CI-AKI may lead to significant morbidity and mortality in high-risk patients. Statins are increasingly recognised as effective in preventing CI-AKI. In this review, we reviewed the literature on statin use for prophylaxis of CI-AKI, its potential benefits, and adverse effects. The aim of the present review was to reveal gaps and discrepancies in the available literature, and to identify areas for future research.

Methods: We searched PubMed for articles published up to 2018, using keywords including: "Statins AND contrast-induced kidney injury", "3-hydroxy3-methyl-glutaryl-CoA reductase inhibitors AND contras-induced kidney injury", and "HMGCoA reductase inhibitors AND contrast induced nephropathy".

Results: Various trials and reviews have yielded promising results in terms of statin efficacy. However, conflicting results and a lack of homogeneity in the protocols of these trials have limited the applicability of statin-based therapy in clinical practice. Despite the development of persistent renal disease, causing

the reported beneficial therapeutic effects of shortterm high-dosage statin use in preventing CI-AKI, statin therapy is not yet the standard prophylactic regimen due to widespread heterogeneity in the clinical trials.

Conclusion: Statin therapy can be used as an adjunct to usual prophylactic measures such as adequate hydration and use of low-volume contrast media. Large well-designed trials on the effects of short-term high-dose statin use in preventing CI-AKI should be conducted, to eliminate any form of discrepancy among results, and to clarify any potential adverse effects.

Hong Kong Med J 2019;25:216-21 https://doi.org/10.12809/hkmj187459

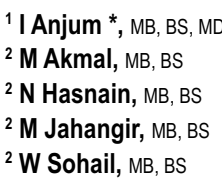

Department of Medicine, California Institute of Behavioral Neurosciences and Psychology, United States

Department of Medicine, Dow Medical College, Karachi, Pakistan

\section{Introduction}

Acute renal failure secondary to contrast-induced acute kidney injury (CI-AKI) is one of the most commonly encountered problems in hospitalised patients. ${ }^{1,2}$ The CI-AKI is generally defined as an increase of at least $0.5 \mathrm{mg} / \mathrm{dL}$ in the plasma creatinine level from the basal value within 24 to 48 hours of contrast exposure. ${ }^{3}$ The rising incidence of CI-AKI in recent decades is concurrent with the increasing use of diagnostic and therapeutic procedures requiring contrast administration, such as coronary angiography (CAG) and percutaneous coronary intervention (PCI). Recent studies have suggested that CI-AKI may lead to the development of persistent renal disease ${ }^{4}$ and is thus a cause of significant morbidity and mortality, ${ }^{5}$ particularly in patients with pre-existing chronic diseases. ${ }^{6}$ Thus, there is an urgent need to design effective prophylactic regimens for CI-AKI to improve the long-term outcomes in patients undergoing such procedures.
The complex pathways involved in the pathogenesis of CI-AKI are not fully understood. Contrast media are known to cause reduced perfusion of renal medulla, owing to an increase in the release of vasoconstrictive mediators and decrease in the vasodilator substances. ${ }^{7,8}$ This leads haemodynamic changes in the renal vasculature that contribute to CI-AKI. Other mechanisms include direct tubular injury by contrast agents and free-radical mediated injury. ${ }^{9}$ Both direct cellular injury and ischaemia act in concert to increase the production of free radicals that can cause cellular injury themselves and thus cause cumulative damage to tubular cells. ${ }^{10}$

An appropriate preventative strategy is the sole means of lowering the risk of contrast-induced nephropathy in high-risk patients, because no intervention is effective once exposure to the contrast medium has already occurred. ${ }^{3}$ Despite extensive research, the best prophylactic approach for acute kidney injury is yet to be discovered. The current recommended strategy is adequate hydration and 
intravascular volume expansion prior to contrast administration. ${ }^{3}$ Although routinely practised, this strategy has not resulted in substantial reduction of CI-AKI; thus, research on optimal prophylactic regimens for $\mathrm{CI}-\mathrm{AKI}$ is necessary.

In recent years, there has been increasing interest in statins for prophylaxis of CI-AKI, although the results to date are controversial. ${ }^{11}$

The aim of the present scoping review was to summarise existing evidence on the efficacy of statins in preventing $\mathrm{CI}-\mathrm{AKI}$, to identify discrepancies and gaps in the available literature, and to recommend areas for future research.

\section{Methods}

We conducted a review of the literature from PubMed for articles published up to May 2018. A variety of keywords were employed including "Statins AND contrast-induced kidney injury", "3-hydroxy3-methyl-glutaryl-CoA reductase inhibitors AND contras-induced kidney injury", "HMGCoA reductase inhibitors AND contrast-induced kidney injury", "Statins AND contrast-induced nephropathy", "3-hydroxy-3-methyl-glutarylCoA reductase inhibitors AND contrast-induced nephropathy", "HMG-CoA reductase inhibitors AND contrast induced nephropathy", "Statins AND CI-AKI", "HMG-CoA reductase inhibitors AND CI-AKI", and "3-hydroxy-3-methyl-glutaryl-CoA reductase inhibitors AND CI-AKI". No language restriction was placed. The abstracts of all the articles were reviewed to assess their relevance to the aims of our study. Our review was conducted in accordance with the PRISMA Extension for Scoping Reviews. ${ }^{12}$

\section{Discussion}

In the past decade, there has been growing interest in discovering the renoprotective effects of statins. The cholesterol-lowering properties of statins are well recognised, supporting their widespread use for the prevention of cardiovascular diseases. ${ }^{13}$ However, studies have also revealed a number of pleiotropic effects of statins which are not directly related to lipid metabolism. ${ }^{14}$ Though the exact mechanism has not been discovered, it has been speculated that statins act by modulation of immune and inflammatory responses, reduction of oxidative stress, prevention of plaque rupture, and improvement in endothelial function. ${ }^{15,16}$ Since aberrations in these responses are thought to be responsible for CI-AKI, statins can potentially be used to effectively prevent CI-AKI.

The efficacy of statins in preventing CI-AKI has been demonstrated in various trials; however, results have been contradictory. One of the earliest metaanalyses by Zhang et $\mathrm{al}^{17}$ showed no statistically significant benefits (relative risk $[R R]=0.76$ ) of

\section{他汀類藥物在預防造影劑引起的急性腎損傷中的 作用：範圍審查}

Ibrar Anjum 、 Manahil Akmal 、 Nimra Hasnain 、

Maha Jahangir - Wafa Sohail

背景：繼發於造影劑引起的急性腎損傷（CI-AKI）的急性腎功能衰竭 是住院患者中其中一個最常見的問題。CI-AKI可導致持續性腎病, 造 成高風險患者的顯著發病率和死亡率。他汀類藥物逐漸被認為可有效 預防CI-AKI。本綜述回顧有關他汀類藥物對預防CI-AKI的潛在益處和 不良反應的文獻。本回顧旨在揭示現有文獻中的差距和差異, 並確定 未來研究的領域。

方法：我們在PubMed搜索截至2018年發表的文章, 並使用關鍵詞 包括：“Statins AND contrast-induced kidney injury”、“3-hydroxy-3methyl-glutaryl-CoA reductase inhibitors AND contras-induced kidney injury”以及“HMG-CoA reductase inhibitors AND contrast induced nephropathy”。

結果：多篇試驗和回顧文章在他汀類藥物療效方面報告可喜的結果。 然而, 這些試驗方案中相互矛盾的結果和缺乏同質性限制了他汀類藥 物治療在臨床實踐中的適用性。儘管報導短期高劑量他汀類藥物用於 預防CI-AKI的有益治療效果, 但由於臨床試驗中廣泛的異質性, 他汀 類藥物治療尚未成為標準預防方案。

結論：他汀類藥物治療可作為常規預防措施的輔助手段, 如充分補水 和使用低容量造影劑。建議進行大型精心設計的短期高劑量他汀類藥 物CI-AKI預防方案試驗, 以消除不同結果之間任何形式的差異, 並澄 清任何潛在的不良反應。

statin pretreatment in preventing CI-AKI. The only significant difference between the treatment arm and the control arm in that study was serum creatinine levels, which were slightly more elevated in patients treated with statins. However, this meta-analysis included only four trials and a total of 752 subjects, increasing the likelihood of bias and rendering the results of the analysis inconclusive. In contrast, several meta-analyses, with greater numbers of trials and patients included, have yielded promising results with pre-procedural statin administration. ${ }^{18-20}$

A recent meta-analysis by $\mathrm{Li}$ et $\mathrm{al}^{21}$ that included 21 randomised controlled trials and 7746 patients observed a significant decrease $(R R=0.57)$ in the likelihood of CI-AKI with statin pretreatment in patients undergoing CAG and PCI. This analysis had some major strengths over previous analyses; for example, the risk of bias in all included studies was gauged using Cochrane Collaboration's tool. This contrasts with earlier analyses ${ }^{22,23}$ which used the Jadad scoring system. ${ }^{24}$ The Jadad scoring system has several inherent shortcomings; for example, it does not account for bias arising from the absence of allocation concealment, which limits its reliability for the assessment of methodological quality of trials.

Another major strength of the meta-analysis by Li et $\mathrm{a}^{21}$ was the use of subgroup analysis to discover 
differences in the effectiveness of statin use with respect to statin type, dosages, duration of therapy, pre-existing diseases, hydration protocols, and definition of CI-AKI. Although the need for better quality trials was emphasised, the authors of that study recommended the use of statins in patients undergoing CAG and PCI, particularly those at high risk of CI-AKI.

The most recent review on statins by Verdoodt et $\mathrm{al}^{11}$ concluded that, although statins are useful for the prevention of cardiovascular diseases, their effectiveness in acute kidney injury and chronic kidney disease (CKD) remains unclear. There is a considerable lack of homogeneity in trials of statin use for preventing CI-AKI, not only in the protocols and methodological designs, but also in the clinical settings in which contrast administration was required. This has limited the validity and reliability of the results of the meta-analyses that have been performed using these trials.

\section{Type, dosage, duration, and timing of statin therapy}

There are inadequate data regarding the differences in the efficacy of different types of statins, mainly because many trials have only compared one type of statin versus placebo. Li et $\mathrm{al}^{21}$ used subgroup analysis to determine discrepancies among different statins and found that the prophylactic effect of statins in CI-AKI was seen irrespective of the type of statin used. Most studies have used one of three statins: rosuvastatin, atorvastatin, or simvastatin. Of these, an appreciable amount of evidence exists for rosuvastatin and atorvastatin, but data on simvastatin are insufficient to draw any reliable conclusions. Liu et $\mathrm{a}^{22}$ did not find any difference in the incidence of CI-AKI between patients treated with rosuvastatin and atorvastatin. However, in another experimental study, rosuvastatin was found to yield better outcomes than simvastatin and atorvastatin. ${ }^{25}$ Yang et $\mathrm{al}^{26}$ performed a meta-analysis of five randomised controlled trials including a total of 4045 patients that compared the effects of rosuvastatin versus placebo and discovered that rosuvastatin administration prior to cardiac catheterisation caused a notable decrease in the risk of CI-AKI. However, since only one type of statin was used, comparisons could not be made among different types of statins. Current evidence indicates that all statin types have similar effects in the prophylaxis of CI-AKI.

Statins have consistently demonstrated higher efficacy at higher doses. In the meta-analysis by Li et al, ${ }^{21}$ compared with lower-dose statin, highdose statins were associated with an absolute risk reduction of $63 \%$ although the quality of the evidence was reportedly low. Cheungpasitporn et $\mathrm{al}^{23}$ reported that only three out of 13 trials compared high-versus low-dose statins; however, all trials that showed a decreased risk of CI-AKI with statin pretreatment used moderately high-dose statins. Another metaanalysis that evaluated the statin efficacy in patients undergoing CAG found high-dose statins to be more effective. ${ }^{27}$ However, significant heterogeneity in the protocols of the trials and differing baseline characteristics of the patients render it difficult to decide on a single-best dosing regimen. This highlights the need to ensure homogeneity in the protocols of the future trials, so that more reliable conclusions can be drawn.

Timing and duration of statin therapy differed markedly across the trials. Most meta-analyses did not perform subgroup analyses based on these parameters. Li et $\mathrm{al}^{21}$ studied the effect of shortterm statin treatment on the incidence of CI-AKI, but the duration of therapy that qualified as 'shortterm' was not specified. This was also the case with the meta-analysis by Ukaigwe et al, ${ }^{27}$ which had several strengths but did not elaborate on the timing and duration of statin therapy separately. A metaanalysis by Barbieri et $\mathrm{al}^{20}$ which demonstrated half the risk of CI-AKI in the statin group versus the control group specified the duration of statin therapy (12 hours to 3 days); however, the effect of therapy duration on outcomes was not determined.

\section{Influence of hydration protocols and volume and strength of contrast media}

Most studies have shown that the combination of hydration with statins yields optimal results. Verdoodt et $\mathrm{al}^{11}$ concluded that adequate intravenous hydration with iso-osmolar crystalloids is the best preventative measure for acute coronary syndrome or for patients undergoing CAG and PCI. The metaanalysis by Barbieri et $\mathrm{al}^{20}$ reported the administration of periprocedural hydration in all but one study, in which hydration was administered only in patients with serum creatinine level $<1.5 \mathrm{mg} / \mathrm{dL}$ or creatinine clearance $>60 \mathrm{~mL} / \mathrm{min} .{ }^{28}$ Quintavalle et $\mathrm{al}^{29}$ primarily used sodium bicarbonate solution for hydration. In contrast, most other studies have reported the use of isotonic saline instead of sodium bicarbonate solution for hydration, with ${ }^{30-32}$ or without ${ }^{33-36}$ $\mathrm{N}$-acetylcysteine. However, a recent large-scale multi-centre prospective randomised trial (the 'Acetylcysteine for Contrast-induced nephropathy Trial $^{37}$ ) demonstrated the ineffectiveness of $\mathrm{N}$ acetylcysteine in cases of CI-AKI. ${ }^{38,39}$

High-volume contrast media $(>100 \mathrm{~mL})$ are associated with a particularly high risk of adverse renal events and the current recommendation is the use of low-volume contrast media. ${ }^{40}$ However, Li et $\mathrm{al}^{21}$ found that the benefit of statin therapy was observed even in patients administered with contrast media volumes as high as $\geq 140 \mathrm{~mL}$, although the evidence was of moderate quality for this subgroup of patients. 
Statins were found to be useful in cases of both low-osmolar $(R R=0.42)$ and iso-osmolar $(R R=0.59)$ contrast media. The quality of evidence for both subgroups was high. ${ }^{21}$ An earlier meta-analysis by Barbieri et $\mathrm{al}^{20}$ also demonstrated the efficacy of statins in CI-AKI which was independent of the strength of contrast media.

\section{Measures of assessment}

To assess the efficacy of statin use, different studies assessed renal function via different measures. For example, a number of studies ${ }^{28,32,41,42}$ used an increase in serum creatinine of $\geq 0.5 \mathrm{mg} / \mathrm{dL}$ or $>25 \%$ from baseline within $48 \mathrm{~h}$ after procedure, whereas others $^{31,32,43}$ used the same criteria but within $72 \mathrm{~h}$. Acikel et $\mathrm{al}^{42}$ and Toso et $\mathrm{al}^{44}$ assessed renal function by an increase in serum creatinine of $\geq 0.5 \mathrm{mg} / \mathrm{dL}$ within 5 days after contrast exposure. Those findings suggest significantly lower postprocedural serum creatinine level among patients in the statin-use group than among those in the control group $(\mathrm{P}<0.0001) .{ }^{21} \mathrm{~A}$ few studies have excluded patients on the criteria of serum creatinine level of $>3 \mathrm{mg} / \mathrm{dL}$. Quintavalle et $\mathrm{a}^{29}$ reported the incidence of CI-AKI on the basis of increases in serum cystatin $C$ $\geq 10 \%$ from baseline within 24 hours after contrast exposure. Creatinine clearance is another parameter used to enrol patients in different studies, for example with a creatinine clearance of $<60 \mathrm{~mL} / \mathrm{min}^{8,21,22}$ or $<70 \mathrm{~mL} / \mathrm{min} .{ }^{32}$ A meta-analysis indicated that, in some studies, postprocedural estimated glomerular filtration rate was higher among patients in the statin-use group than among those in the control group $(\mathrm{P}=0.001){ }^{21}$ However, no restrictions on the basis of renal function were imposed by Li et al. ${ }^{31}$

Most articles, including meta-analyses and reviews, have not commented on whether different effects of statins were observed in different populations. However, a meta-analysis conducted by Mao and Huang ${ }^{19}$ included trials consisting of Caucasian and Asian populations. The authors reported that the effect of statins in both groups was equally significant. ${ }^{19} \mathrm{~A}$ meta-analysis by $\mathrm{Li}$ et $\mathrm{al}^{21}$ was the first to report better outcomes in East Asian and statin-naïve patients. Another meta-analysis that performed subgroup analysis for different populations found that there were no differences in the efficacy of statins among different racial populations, suggesting that genetic polymorphisms may not have an important role in determining the efficacy of statins in CI-AKI. ${ }^{27}$

\section{Effect of underlying diseases/risk factors on statin efficacy}

Most previous studies recruited patients who already had some underlying disease or precipitating risk factor for CI-AKI. Advanced age, type and volume of contrast, pre-existing disease such as congestive heart failure and CKD, and haemodynamic instability are reportedly more likely to influence the development of CI-AKI. ${ }^{11}$ Chyou et $\mathrm{a}^{45}$ demonstrated that increased age, diabetes mellitus, acute coronary syndrome, and CKD are the factors responsible for precipitating the hazard for contrast-induced nephropathy. Further, Chung et $\mathrm{al}^{46}$ confirmed that there is a $13 \%$ increased risk of developing severe renal failure with statin treatment among the highrisk population. Quintavalle et $\mathrm{a}^{29}$ found lower rates of CI-AKI occurrence in patients with CKD, whereas Toso et $\mathrm{al}^{44}$ did not find decrease in the occurrence of CI-AKI in patients with existing CKD with highdose atorvastatin; however, high-dose rosuvastatin was effective in these patients. ${ }^{44}$

The study by Li et al ${ }^{21}$ was the first to assess the benefits of statin therapy in patients with diabetes mellitus, acute coronary syndrome, CKD, or congestive heart failure and those requiring highervolume contrast media. The authors observed that statins proved useful regardless of these risk factors, although the quality of evidence varied from low to high. The authors found that the risk of CI-AKI was $4.4 \%$ in the diabetes mellitus subgroup compared with $6.5 \%$ in control group $(\mathrm{RR}=0.70){ }^{21}$ The overall risk reduction was $5.0 \%$ in the statin pretreatment arm compared with $8.4 \%$ in the control arm $(R R=0.61){ }^{21}$ Thus, the results of these meta-analysis indicate that statins are effective prophylactic agents for CI-AKI even in patients with risk factors such as CKD and diabetes mellitus.

\section{Adverse effects}

As most trials reviewed did not have a long-term follow-up, the frequency of adverse events with statin use for prophylaxis of CI-AKI is not accurately known. ${ }^{43} \mathrm{~A}$ recent updated review published by Verdoodt et $\mathrm{al}^{11}$ was sceptical of the beneficial effects of statins owing to the wide range of potential adverse effects. A large retrospective cohort study from Taiwan found that high-efficacy statins increased the risk of severe renal failure by $13 \%$ compared with low-efficacy statins, such as lovastatin, pravastatin, simvastatin, and fluvastatin. ${ }^{46}$ Myopathy is a common adverse effect of statin use and its risk is further increased by concomitant CKD. Statin-induced myopathy clinically manifests as a mild increase in creatinine kinase levels, myalgia, myositis, and rhabdomyolysis. The incidences of these effects have not been reported in most trials that have focused on CI-AKI. Thompson et $\mathrm{a}^{47}$ reviewed data from two databases and revealed that the incidence of myalgia ranged from $6 \%$ to $14 \%$ in one database and from $19 \%$ to $25 \%$ in the other. However, the clinical trials rarely report on the incidence of myalgia. In almost all trials and meta-analyses that explored the efficacy of statin use for the prevention of CI-AKI, adverse effects of statin use were not documented. 
This highlights the need for long-term follow-up of patients undergoing statin prophylaxis, so that the potential adverse effects of statin use can be clarified.

\section{Conclusion}

Although several studies have implied beneficial therapeutic effects of short-term high-dose statin use in preventing CI-AKI, statin therapy is not yet the standard prophylactic regimen. Widespread heterogeneity in clinical trials has resulted in inconclusive and contradictory findings regarding the efficacy of statin use for preventing CI-AKI. Large and well-designed trials with more homogeneous protocols should be conducted to minimise discrepancies among the results. Statin therapy can be used as an adjunct to usual prophylactic measures such as adequate hydration and use of low-volume contrast media. However, further controlled trials are required to clarify the harmful potential of statin use in the context of CI-AKI, before this treatment is adopted in clinical practice.

\section{Author contributions}

All authors had full access to the data, contributed to the study, approved the final version for publication, and take responsibility for its accuracy and integrity.

Concept and design of study: I Anjum.

Acquisition of data: M Akmal, N Hasnain, M Jahangir.

Analysis or interpretation of data: W Sohail.

Drafting of the manuscript: M Akmal, N Hasnain, M Jahangir. Critical revision for important intellectual content: I Anjum, W Sohail.

\section{Conflicts of interest}

All authors have declared no conflicts of interest.

\section{Funding/support}

This research received no specific grant from any funding agency in the public, commercial, or not-for-profit sectors.

\section{References}

1. Nash K, Hafeez A, Hou S. Hospital-acquired renal insufficiency. Am J Kidney Dis 2002;39:930-6.

2. McCullough PA, Wolyn R, Rocher LL, Levin RN, O’Neill WW. Acute renal failure after coronary intervention: incidence, risk factors, and relationship to mortality. Am J Med 1997;103:368-75.

3. Ozkok S, Ozkok A. Contrast-induced acute kidney injury: a review of practical points. World J Nephrol 2017;6:86-99.

4. Maioli M, Toso A, Leoncini M, Gallopin M, Musilli N, Bellandi F. Persistent renal damage after contrast-induced acute kidney injury: incidence, evolution, risk factors, and prognosis. Circulation 2012;125:3099-107.

5. Rudnick M, Feldman H. Contrast-induced nephropathy: what are the true clinical consequences? Clin J Am Soc Nephrol 2008;3:263-72.

6. Parfrey PS, Griffiths SM, Barrett BJ, et al. Contrast materialinduced renal failure in patients with diabetes mellitus, renal insufficiency, or both. A prospective controlled study. N Engl J Med 1989;320:143-9.

7. Sendeski M, Patzak A, Pallone TL, Cao C, Persson AE, Persson PB. Iodixanol, constriction of medullary descending vasa recta, and risk for contrast mediuminduced nephropathy. Radiology 2009;251:697-704.

8. Liu ZZ, Schmerbach K, Lu Y, et al. Iodinated contrast media cause direct tubular cell damage, leading to oxidative stress, low nitric oxide, and impairment of tubuloglomerular feedback. Am J Physiol Renal Physiol 2014;306:F864-72.

9. Katzberg RW. Contrast medium-induced nephrotoxicity: which pathway? Radiology 2005;235:752-5.

10. Geenen RW, Kingma HJ, van der Molen AJ. Pathophysiology of contrast-induced acute kidney injury. Interv Cardiol Clin 2014;3:363-7.

11. Verdoodt A, Honore PM, Jacobs R, et al. Do statins induce or protect from acute kidney injury and chronic kidney disease: an update review in 2018. J Transl Int Med 2018;6:21-5.

12. Tricco AC, Lillie E, Zarin W, et al. PRISMA Extension for Scoping Reviews (PRISMA-ScR): checklist and explanation. Ann Intern Med 2018;169:467-73.

13. Davies JT, Delfino SF, Feinberg CE, et al. Current and emerging uses of statins in clinical therapeutics: a review. Lipid Insights 2016;9:13-29.

14. Liao JK, Laufs U. Pleiotropic effects of statins. Annu Rev Pharmacol Toxicol 2005;45:89-118.

15. Hernández-Perera O, Pérez-Sala D, Navarro-Antolin J, et al. Effects of the 3-hydroxy-3-methylglutaryl-CoA reductase inhibitors, atorvastatin and simvastatin, on the expression of endothelin-1 and endothelial nitric oxide synthase in vascular endothelial cells. J Clin Invest 1998;101:2711-9.

16. Al-Otaibi KE, Al Elaiwi AM, Tariq M, Al-Asmari AK. Simvastatin attenuates contrast-induced nephropathy through modulation of oxidative stress, proinflammatory myeloperoxidase, and nitric oxide. Oxid Med Cell Longev 2012;2012:831748.

17. Zhang L, Zhang L, Lu Y, et al. Efficacy of statin pretreatment for the prevention of contrast-induced nephropathy: a meta-analysis of randomised controlled trials. Int J Clin Pract 2011;65:624-30.

18. Liu LY, Liu Y, Wu MY, Sun YY, Ma FZ. Efficacy of atorvastatin on the prevention of contrast-induced acute kidney injury: a meta-analysis. Drug Des Devel Ther 2018;12:437-44.

19. Mao S, Huang S. Statins use and the risk of acute kidney injury: a meta-analysis. Ren Fail 2014;36:651-7.

20. Barbieri L, Verdoia M, Schaffer A, Nardin M, Marino P, De Luca G. The role of statins in the prevention of contrast induced nephropathy: a meta-analysis of 8 randomized trials. J Thromb Thrombolysis 2014;38:493-502.

21. Li H, Wang C, Liu C, Li R, Zou M, Cheng G. Efficacy of short-term statin treatment for the prevention of contrast-induced acute kidney injury in patients undergoing coronary angiography/percutaneous coronary intervention: a meta-analysis of 21 randomized controlled trials. Am J Cardiovasc Drugs 2016;16:201-19.

22. Liu YH, Liu Y, Duan CY, et al. Statins for the prevention of contrast-induced nephropathy after coronary angiography/percutaneous interventions: a meta-analysis of randomized controlled trials. J Cardiovasc Pharmacol Ther 2015;20:181-92.

23. Cheungpasitporn W, Thongprayoon C,Kittanamongkolchai 
W, et al. Periprocedural effects of statins on the incidence of contrast-induced acute kidney injury: a systematic review and meta-analysis of randomized controlled trials. Ren Fail 2015;37:664-71.

24. Jadad AR, Moore RA, Carroll D, et al. Assessing the quality of reports of randomized clinical trials: is blinding necessary? Control Clin Trials 1996;17:1-12.

25. Ferreira TS, Lanzetti M, Barroso MV, et al. Oxidative stress and inflammation are differentially affected by atorvastatin, pravastatin, rosuvastatin, and simvastatin on lungs from mice exposed to cigarette smoke. Inflammation 2014;37:1355-65.

26. Yang Y, Wu YX, Hu YZ. Rosuvastatin treatment for preventing contrast-induced acute kidney injury after cardiac catheterization: a meta-analysis of randomized controlled trials. Medicine (Baltimore) 2015;94:e1226.

27. Ukaigwe A, Karmacharya P, Mahmood M, et al. Metaanalysis on efficacy of statins for prevention of contrastinduced acute kidney injury in patients undergoing coronary angiography. Am J Cardiol 2014;114:1295-302.

28. Patti G, Ricottini E, Nusca A, et al. Short-term, high-dose atorvastatin pretreatment to prevent contrast-induced nephropathy in patients with acute coronary syndromes undergoing percutaneous coronary intervention (from the ARMYDA-CIN [atorvastatin for reduction of myocardial damage during angioplasty-contrast-induced nephropathy] trial. Am J Cardiol 2011;108:1-7.

29. Quintavalle C, Fiore D, De Micco F, et al. Impact of a high loading dose of atorvastatin on contrast-induced acute kidney injury. Circulation 2012;126:3008-16.

30. Leoncini M, Toso A, Maioli M, Tropeano F, Villani S, Bellandi F. Early high-dose rosuvastatin for contrastinduced nephropathy prevention in acute coronary syndrome: Results from the PRATO-ACS Study (Protective Effect of Rosuvastatin and Antiplatelet Therapy On contrast-induced acute kidney injury and myocardial damage in patients with Acute Coronary Syndrome). J Am Coll Cardiol 2014;63:71-9.

31. Li W, Fu X, Wang Y, et al. Beneficial effects of highdose atorvastatin pretreatment on renal function in patients with acute ST-segment elevation myocardial infarction undergoing emergency percutaneous coronary intervention. Cardiology 2012;122:195-202.

32. Özhan H, Erden I, Ordu S, et al. Efficacy of short-term high-dose atorvastatin for prevention of contrast-induced nephropathy in patients undergoing coronary angiography. Angiology 2010;61:711-4.

33. Jia X, Fu X, Zhang J, et al. Comparison of usefulness of simvastatin $20 \mathrm{mg}$ versus $80 \mathrm{mg}$ in preventing contrastinduced nephropathy in patients with acute coronary syndrome undergoing percutaneous coronary intervention. Am J Cardiol 2009;104:519-24.

34. Yun KH, Lim JH, Hwang KB, et al. Effect of high dose rosuvastatin loading before percutaneous coronary intervention on contrast-induced nephropathy. Korean Circ J 2014;44:301-6.

35. Abaci O, Arat Ozkan A, Kocas C, et al. Impact of rosuvastatin on contrast-induced acute kidney injury in patients at high risk for nephropathy undergoing elective angiography. Am J Cardiol 2015;115:867-71.

36. Qiao B, Deng J, Li Y, Wang X, Han Y. Rosuvastatin attenuated contrast-induced nephropathy in diabetes patients with renal dysfunction. Int J Clin Exp Med 2015;8:2342-9.

37. ACT Investigators. Acetylcysteine for prevention of renal outcomes in patients undergoing coronary and peripheral vascular angiography: main results from the randomized Acetylcysteine for Contrast-induced nephropathy Trial (ACT). Circulation 2011;124:1250-9.

38. Akyuz S, Yaylak B, Altay S, Kasikcioglu H, Cam N. The role of statins in preventing contrast-induced acute kidney injury: a narrative review. Angiology 2015;66:701-7.

39. O'Sullivan S, Healy DA, Moloney MC, Grace PA, Walsh $\mathrm{SR}$. The role of $\mathrm{N}$-acetylcysteine in the prevention of contrast-induced nephropathy in patients undergoing peripheral angiography: a structured review and metaanalysis. Angiology 2013;64:576-82.

40. McCullough PA. Contrast-induced acute kidney injury. J Am Coll Cardiol 2008;51:1419-28.

41. Jo SH, Koo BK, Park J, et al. Prevention of radiocontrast medium-induced nephropathy using short-term high-dose simvastatin in patients with renal insufficiency undergoing coronary angiography (PROMISS) trial-a randomized controlled study. Am Heart J 2008;155:499.e1-8.

42. Acikel S, Muderrisoglu H, Yildirir A, et al. Prevention of contrast-induced impairment of renal function by shortterm or long-term statin therapy in patients undergoing elective coronary angiography. Blood Coagul Fibrinolysis 2010;21:750-7.

43. Han Y, Zhu G, Han L, et al. Short-term rosuvastatin therapy for prevention of contrast-induced acute kidney injury in patients with diabetes and chronic kidney disease. J Am Coll Cardiol 2014;63:62-70.

44. Toso A, Maioli M, Leoncini $M$, et al. Usefulness of atorvastatin $(80 \mathrm{mg})$ in prevention of contrast-induced nephropathy in patients with chronic renal disease. Am J Cardiol 2010;105:288-92.

45. Chyou AC, Thodge A, Feldman DN, Swaminathan RV. Statins in the prevention of contrast-induced nephropathy. Curr Treat Options Cardiovasc Med 2015;17:375.

46. Chung $\mathrm{YH}$, Lee YC, Chang $\mathrm{CH}$, Lin MS, Lin JW, Lai MS. Statins of high versus low cholesterol-lowering efficacy and the development of severe renal failure. Pharmacoepidemiol Drug Saf 2013;22:583-92.

47. Thompson PD, Clarkson P, Karas RH. Statin-associated myopathy. JAMA 2003;289:1681-90. 